\title{
Text Dependent Speaker Identification using Hidden Markchov Model and Mel Frequency Cepstrum Coefficient
}

\author{
Mohd. Manjur Alam \\ Lecturer \\ Dept. of CSE \\ BGC Trust University \\ Bangladesh
}

\author{
Md. Salah Uddin \\ Chowdurye \\ Assistant Professor \\ Dept. of CSE \\ BGC Trust University \\ Bangladesh
}

\author{
Niaz Uddin Mahmud \\ Lecturer \\ Dept. of CSE \\ BGC Trust University \\ Bangladesh
}

\begin{abstract}
Speaker identification is a biometric process. The objective of speaker identification is to extract, characterize and recognize the information about speaker identity. Speaker Recognition technology has recently been used in a vast number of commercial areas successfully such as in voice based biometrics; voice controlled appliances, security control for confidential information, remote access to computers and many more interesting areas. A speaker identification system has two phases which are the training phase and the testing phase. Feature extraction is the first step for each phase in speaker recognition. Many algorithms are used for feature extraction. In this work, the Mel Frequency Cepstrum Coefficient (MFCC) feature has been used for designing a text dependent speaker identification system. In the identification phase, the existing reference templates are compared with the unknown voice input. In this thesis, Hidden Markov Model (HMM) method is used as the training/recognition algorithm which makes the final decision about the specification of the speaker by comparing unknown features to all models in the database and selecting the best matching model. i, e. the highest scored model. The speaker who obtains the highest score is selected as the target speaker.
\end{abstract}

\section{Keywords}

Mel Frequency Cepstrum Coefficient (MFCC), Hidden Markchov Model (HMM), Speaker Identification (SI), Fast Fourier Transform (FFT).

\section{INTRODUCTION}

Speaker identification system is divided into text dependent and text independent recognition systems [1]. In text dependent recognition systems, systems know the text spoken by person. Fixed or prompted phrases or texts are used by the speakers. Same text must be used for enrollment and verification. They generally are more accurate and improve system performance as system has knowledge of spoken text. In text independent recognition, system does not know the text to be spoken by a speaker. Text entered during enrollment and test is different. It is more flexible but also more difficult. Recognizing natural speech is a challenging task as we are unable to see the speech signals. Amplitude, pitch, phonetic emphasis etc. are the various speech parameters that are used in speech recognition systems. This identification is textdependant.
The human speech contains numerous discriminative features used to identify speakers [2]. Speech contains significant energy from zero frequency up to around $5 \mathrm{kHz}$. The objective of automatic speaker identification is to extract, characterize and recognize the information about speaker identity. Anatomical structure of the vocal tract is unique for every person and hence the voice information available in the speech signal can be used to identify the speaker. Recognizing a person by her/his voice is known as speaker recognition. Since differences in the anatomical structure are an intrinsic property of the speaker, voice comes under the category of biometric identity. Using voice for speaker identification has several advantages. One of the major advantages is remote person authentication. The property of speech signal changes markedly as a function of time. To study the spectral properties of speech signal the concept of time varying Fourier representation is used. However, the temporal properties of speech signal such, as energy, zero crossing, correlation etc are assumed constant over a short period. Its characteristics are short-time stationary .Therefore, using hamming window, Speech signal is analyzed into a number of blocks of short duration so that normal Fourier transform can be used. In this thesis the most important thing is to extract the feature from the speech signal. The speech feature extraction in a categorization problem is about reducing the dimensionality of the input-vector while maintaining the discriminating power of the signal. As we know from the above fundamental formation of speaker identification that the number of training and test vector needed for the classification problem grows exponential with the dimension of the given input vector, so we need feature extraction. But extracted feature should meet some criteria while dealing with the speech signal.

Such as:

- $\quad$ Easy to measure extracted Speech features.

- Distinguish between speakers while being lenient of intra speaker variability's.

- It should not be susceptible to mimicry.

- It should show little fluctuation from one speaking environment to another.

- It should be stable over time.

- It should occur frequently and naturally in speech. 
In this work, the Mel frequency Cepstrum Coefficient (MFCC) feature has been used for designing a text dependent speaker identification system. A speaker recognition system consists of two phases which is the training phase and the testing phase. In the training phase, the speaker voices are recorded and processed in order to generate the model to store in the database. While, in the identification phase, the existing reference templates are compared with the unknown voice input. The identification is done using HMM. The code is developed in the MATLAB environment and performs the identification satisfactorily.

\section{PHASES OF SPEAKER} IDENTIFICATION

Like any other pattern recognition systems, speaker recognition systems also involve two phases namely, training and testing [2]. Training is the process of familiarizing the system with the voice characteristics of the speakers registering. Testing is the actual recognition task. We make the final decision about the identity of the speaker by comparing unknown features to all models in the database and selecting the best matching model. i, e. the highest scored model. This is done using HMM.

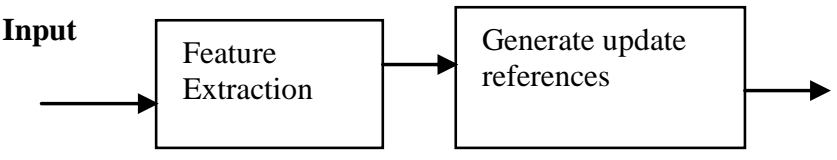

Fig 1:The block diagram of the training phase

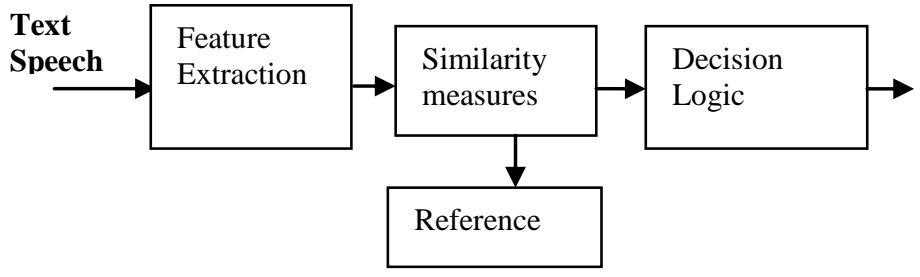

Fig 2: The block diagram of the testing phase

\section{SPEECH FEATURE EXTRACTION}

In this thesis the most important thing is to extract the feature from the speech signal. Mel Frequency Cepstral Coefficients (MFCC) are used to extract features from the speech signal and compare the unknown speaker with the exits speaker in the database. MFCCs are based on the known variation of the human ear's critical bandwidths with frequency; filters spaced linearly at low frequencies and logarithmically at high frequencies have been used to capture the phonetically important characteristics of speech. This is expressed in the mel-frequency scale, which is linear frequency spacing below $1000 \mathrm{~Hz}$ and a logarithmic spacing above $1000 \mathrm{~Hz}$. Fig3. below shows the complete pipeline of Mel Frequency Cepstral Coefficients

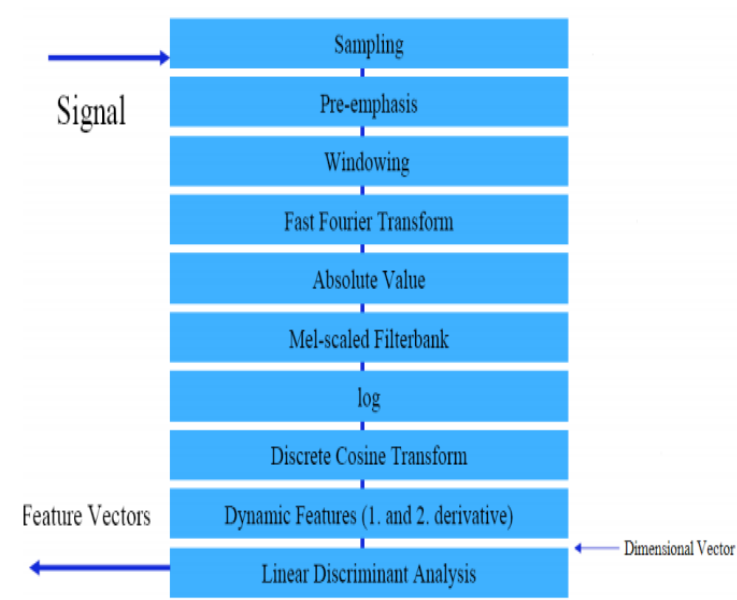

Fig 3: Mel Frequency Cepstral Coefficients

\subsection{Framing and Windowing}

As shown in the figure (Fig4) the speech signal is slowly varying over time and it is called quasi stationery. When the speech signal is examined over a short period of time such as 5 to 100 milliseconds, the signal is reasonably stationery, and therefore these signals are examine in short time segment.

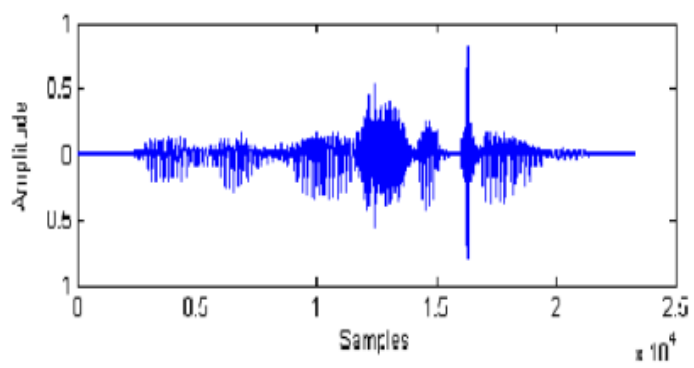

Fig 4: Framing and Windowing

\subsection{Hamming Window}

Hamming window is also called the raised cosine window.

\subsection{Fast Fourier Transform}

FFT is a very important mathematical tool.FFT algorithms are the discrete time sequence $\mathrm{x}(\mathrm{n})$ is transformed into corresponding discrete frequency sequence $\mathrm{X}[\mathrm{k}]$. The FFT is a fast algorithm to implement the Discrete Fourier Transform (DFT), which is defined on the set of $N$, samples $\{x n\}$, as follow [3]:

$X_{k}=\sum_{n=0}^{N-1} x_{n} e^{-j 2 \pi k n / N} \quad k=0,1,2, \ldots N$

When Xk's are generally complex numbers and we only consider their absolute frequencies here. The resulting sequence $\{\mathrm{Xk}\}$ is interpreted as follow: positive frequencies $0 \leq \mathrm{f}<\mathrm{Fs} / 2$ correspond to values $0 \leq \mathrm{n} \leq \mathrm{N} / 2-1$, while negative frequencies $-\mathrm{Fs} / 2<\mathrm{f}<0$ correspond to $\mathrm{N} / 2+1 \leq \mathrm{n} \leq \mathrm{N}-1$. Here, Fs denote the sampling frequency.

\subsection{Mel Frequency Warping}

For each sound with an actual frequency, $\mathrm{f}$, measured in $\mathrm{Hz}$, a subjective frequency is measured on a scale called the "Mel scale" Mel-frequency can be approximated by

$$
\operatorname{Mel}(\mathrm{f})=1127 \ln \frac{f}{100}+1
$$

Where $\mathrm{f}$ in $\mathrm{Hz}$, is the actual frequency of the sound [3] 


\subsection{Cepstrum}

It is defined as the inverse Fourier transform of the logarithm of the magnitude of the Fourier transform; i.e.

cepstrum $=$ iffft $(\log (\mid \mathrm{fft}($ signal $) \mid))$

\subsection{Triangular Filters Bank}

We define a triangular filter-bank with $M$ filters $(m=1$, $2, \ldots, \mathrm{M}$ ) and $\mathrm{N}$ points Discrete Fourier Transform (DFT) $(\mathrm{k}=1,2, \ldots, \mathrm{N})$, where $\mathrm{Hm}[\mathrm{k}]$ is the magnitude (frequency response) of the filter given by[3]:

$H_{m}(k)=\left\{\begin{array}{cc}0, & k<f[m-1] \\ \frac{(k-f[m-1])}{(f[m]-f[m-1]),} & f[m-1] \leq k \leq f[m] \\ \frac{f[m+1]-k)}{f[m+1]-f[m]}, & f[m] \leq k \leq f[m+1] \\ 0, & k>f[m+1]\end{array}\right.$

$f[m]=\left(\frac{N}{F_{s}}\right) \operatorname{Mel}^{-1}\left(\operatorname{Mel}\left(f_{l}\right)+m \frac{\operatorname{Mel}\left(f_{h}\right)-\operatorname{Mel}\left(f_{l}\right)}{M}\right)$

Where Mel (f) is given by (1) and 『Mel『^ (-1) (f_l) is its inverse given by (6)

$\mathrm{Mel}^{-1}(f)=700\left(\exp \left(\frac{f}{1127}\right)\right.$

Where $\mathrm{f}$ in Mel, is the subjective frequency (Mel-frequency)

\subsection{Calculation of Mfces}

Given the DFT of the input signal, $x[n][3]$

$X_{a}[k]=\sum_{n=0}^{N-1} x[n] e^{-\frac{j 2 \pi n k}{N}}, 0 \leq k \leq N$

In most implementations of speech recognition, a short-time Fourier analysis is done first, resulting in a DFT, X_(a ) [K], for the ath frame. Then the values of DFT are weighted by triangular filters. The result is called Mel-frequency power spectrum which is defined:

$$
S[m]=\sum_{k=1}^{N} X_{a}[K]^{2} H_{m}[K], 0<m \leq M
$$

Where $X \_a \llbracket[K] \rrbracket \wedge 2$ is called power spectrum. Finally, a discrete cosine transform (DCT) of the logarithm of S[m] is computed to form the MFCCs as

$$
m f c c[i]=\sum_{m=1}^{M} \log \left[i s[m] \cos \left[i\left(m-\frac{1}{2}\right)\right] \frac{\pi}{M}\right.
$$

Where $\mathrm{L}$ is the number of cepstrum coefficients.

Such filters compute the average spectrum around each center frequency with increasing bandwidths, and they are displayed in Fig 5. Let fland fh be the lowest and highest frequencies of the filter-bank in $\mathrm{Hz}$, Fs the sampling frequency in $\mathrm{Hz}, \mathrm{M}$ the number of filters, and $\mathrm{N}$ the size of the Fast Fourier Transform.

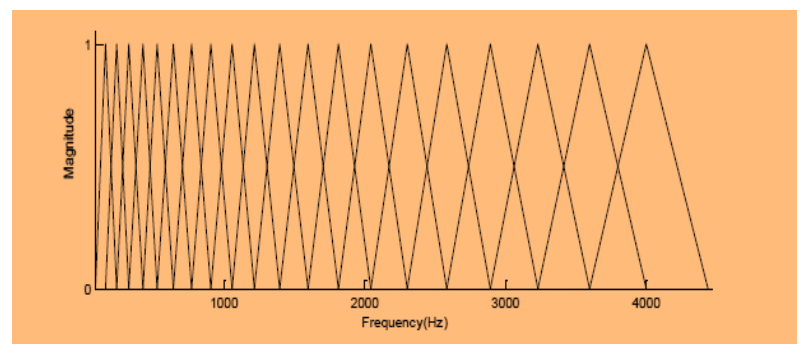

Fig 5: Filter bank for generating Mel-Frequency Cepstrum Coefficients

\section{HMM FOR SPEAKER IDENTIFICATION}

In this thesis, we use the Hidden Markov Model (HMM) method as the training/recognition algorithm [1]. The most flexible and successful approach to speech recognition so far has been HMM. The goal of HMM parameter estimation is to maximize the likelihood of the data under the given parameter setting. There are 3 basic parameters in HMM which is:

1. $\pi$ - The initial state distribution.

2. a- The state-transition probability matrix.

3. B - Observation probability distribution.

In the training phase, a HMM model for each speaker is generated. After $\mathrm{N}$ models have been created, the HMM engine is then ready for speaker identification.

\subsection{Estimating the Parameters}

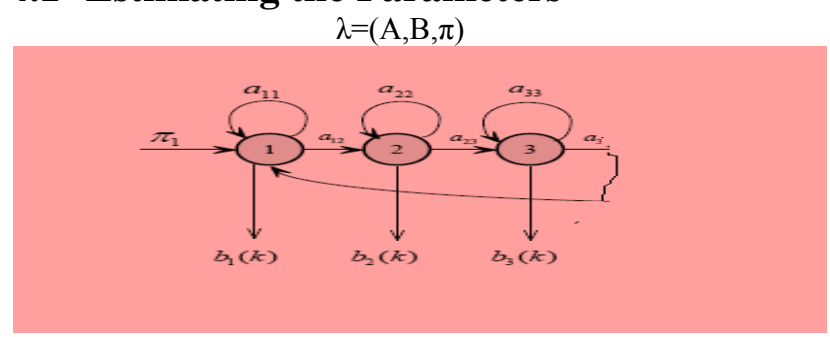

Fig.6: HMM for each speaker

Here same word is uttered for three times, hence the HMM has three states.

The model parameters can be estimated as follows:

$$
\begin{aligned}
\pi_{i} & =\gamma_{1}(i) \\
& =\text { expected number of times in state } \mathrm{i} \\
a_{i j} & =\frac{\sum_{t=1}^{T-1} \xi_{t}(i, j)}{\sum_{t=1}^{T-1} \gamma_{t}(i)}
\end{aligned}
$$

$=$ expected number of transition from state $i$ to state $j$

expected number of transition from state $i$

\subsection{Bi (k) Measurement}

Probabilities from states to observations are measured from matching MFCCs from states to observations. The matching MFCCs are proportional to probability. Here the mfcc of each frame of the training data is compared to that of the testing data with sequence for each utterance.

\subsection{Scores}

For each speaker model, probability score for the unknown observation sequence is computed. The speaker whose model produces the highest probability score and matches the ID claimed is then selected as the client speaker. Hence the total probability is given by:

$\mathrm{P}(\mathrm{X} 1 \mathrm{Q})=\prod_{i=1, j=1}^{n} \mathrm{a}_{\mathrm{ij}} \mathrm{b}_{\mathrm{i}}(\mathrm{k})$

\section{EXPERIMENTAL RESULTS}

MFCCs from five speakers saying "CSE" using Matlab have been generated and recorded for speaker recognition. Each speaker has uttered the same word for three times. Here the duration was 3.0 seconds. The speech was recorded using a sampling rate of $44100 \mathrm{~Hz}$ with 16 bits per sample. The waveform corresponding to the utterance is shown at fig7. Speech waveform sampled at $1600 \mathrm{~Hz}$. 


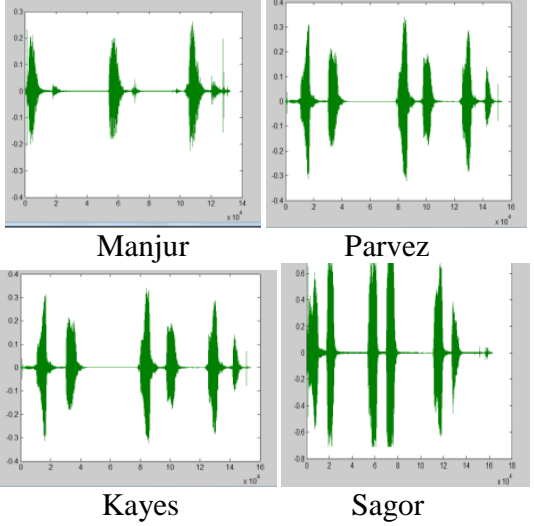

Fig 7: Wave Forms

\subsection{Feature Extraction}

A population of 4 speakers, with 3 utterances(same word) for each speaker have been used. Each utterance was divided into 2210frames (total 6630). The average length of each frame was about 32 milliseconds (256 samples) Then MFCC were calculated for each frame using Matlab. Similarly the features are extracted from testing data. Here the mfcc of each frame of the training data is compared to the of the testing data for each utterance. Only $150 \mathrm{mfccs}$ from mfccs for each speaker: 50 from first utterance, 50 from second one and 50 from third one in training and testing data have been selected.

\subsection{Matching Between Training \& Testing \\ Data}

The mfcc of each frame of the training data is compared to that of the testing data for each utterance. The two mfccs which distance between them within 1.5 are similar. This is done from both the sides.

Matching MFCCS=Total MFCCs - numbers of uncommon MFCCS in training data \& Testing data.

\subsubsection{Conditions}

i. If the numbers of uncommon MFCCS is greater than or equal to the Total MFCCs, then the numbers of matching MFCCS are considered as 1or 2.

ii. if the numbers of matching MFCCS are equal to the total mfccs, then the numbers of matching MFCCS are considered as (Total MFCCs-1).

Table1: Matching between training \& testing data set for each speaker

\begin{tabular}{|l|l|l|l|}
\hline & \multicolumn{3}{|c|}{ Unknown Speaker } \\
\hline & $\begin{array}{l}1^{\text {st }} \\
\text { utterance }\end{array}$ & $\begin{array}{l}\text { 2nd } \\
\text { utterance }\end{array}$ & $\begin{array}{l}3^{\text {rd }} \\
\text { utterance }\end{array}$ \\
\hline Speaker & $\begin{array}{l}\text { matching } \\
\text { mfccs }\end{array}$ & $\begin{array}{l}\text { matching } \\
\text { mfccs }\end{array}$ & $\begin{array}{l}\text { matching } \\
\text { mfccs }\end{array}$ \\
\hline Manjur & 42 & 49 & 49 \\
\hline Parvez & 45 & 1 & 49 \\
\hline Sagor & 27 & 49 & 49 \\
\hline Kayes & 13 & 49 & 49 \\
\hline
\end{tabular}

\subsection{Mi Estimation}

$\pi_{1}=.5, \pi_{2}==.25, \pi_{2}=.25$

\section{$5.4 \mathbf{a}_{\mathrm{ij}}$ Estimation}

Each HMM has three states for three token words and three observations

Hence, $\mathrm{a} 11=\mathrm{a} 12==\mathrm{a} 22==\mathrm{a} 23==\mathrm{a} 33==\mathrm{a} 31==1 / 2=.5$;

\subsection{Bi (k) Measurement}

Probabilities from states to observations are measured from matching MFCCs from states to observations. The matching MFCCs are related to probability. Here the mfcc of each frame of the training data is compared to the of the testing data for each utterance.

bi $(k)=$ Numbers of matching mfccs/total mfccs

From the table 1:

Manjur:

$\mathrm{b} 1(\mathrm{k})=42 / 50=.82, \mathrm{~b} 2(\mathrm{k})=49 / 50=.98, \mathrm{~b} 3(\mathrm{k})=49 / 50=.98$,

Parvez:

$\mathrm{b} 1(\mathrm{k})=45 / 50=.90, \mathrm{~b} 2(\mathrm{k})=01 / 50=.02, \mathrm{~b} 3(\mathrm{k})=49 / 50=.98$,

Sagor:

$\mathrm{b} 1(\mathrm{k})=27 / 50=.54, \mathrm{~b} 2(\mathrm{k})=49 / 50=.98, \mathrm{~b} 3(\mathrm{k})=49 / 50=.98$, Kayes:

$\mathrm{b} 1(\mathrm{k})=13 / 50=.26, \mathrm{~b} 2(\mathrm{k})=49 / 50=.98, \mathrm{~b} 3(\mathrm{k})=49 / 50=.98$,

\subsection{Scores Estimation}

The individual speaker scores are shown on the following tables.

Table 2: Manjur

\begin{tabular}{|llll|}
\hline \hline Observation sequence $(\mathrm{x}):$ & $\mathrm{Q}_{1}$ & $\mathrm{Q}_{2}$ & $\mathrm{Q}_{3}$ \\
State sequence $(\mathrm{Q}):$ & 1 & 2 & 3 \\
State transition probability, $\mathrm{a}_{\mathrm{ij}}:$ & 0.5 & 0.5 & 0.5 \\
Observation probabilities, $\mathrm{b}_{\mathrm{i}}(\mathrm{k}):$ & 0.82 & 0.98 & 0.98 \\
\hline \hline
\end{tabular}

Total probability for Manjur, $\mathrm{P}(\mathrm{X} 1 \mathrm{Q})=.09844$

Table 3:Parvez

\begin{tabular}{|llll|}
\hline \hline Observation sequence $(\mathrm{x}):$ & $\mathrm{Q}_{1}$ & $\mathrm{Q}_{2}$ & $\mathrm{Q}_{3}$ \\
State sequence $(\mathrm{Q}):$ & 1 & 2 & 3 \\
State transition probability, $\mathrm{a}_{\mathrm{ij}}:$ & 0.5 & 0.5 & 0.5 \\
Observation probabilities, $\mathrm{b}_{\mathrm{i}}(\mathrm{k}):$ & 0.90 & 0.02 & 0.98 \\
\hline \hline
\end{tabular}

Total probability for Parvez, $\mathrm{P}(\mathrm{X} 1 \mathrm{Q})=.00220$

Table 4:Sagor

\begin{tabular}{|llll|}
\hline \hline Observation sequence $(\mathrm{x}):$ & $\mathrm{Q}_{1}$ & $\mathrm{Q}_{2}$ & $\mathrm{Q}_{3}$ \\
State sequence $(\mathrm{Q}):$ & 1 & 2 & 3 \\
State transition probability, $\mathrm{a}_{\mathrm{ij}}:$ & 0.5 & 0.5 & 0.5 \\
Observation probabilities, $\mathrm{b}_{\mathrm{i}}(\mathrm{k}):$ & 0.54 & 0.98 & 0.98 \\
\hline \hline
\end{tabular}

Total probability for Sagor, $\mathrm{P}(\mathrm{X} 1 \mathrm{Q})=0.06482$

Table 5:Kayes

\begin{tabular}{llll|}
\hline Observation sequence $(\mathrm{x}):$ & $\mathrm{Q}_{1}$ & $\mathrm{Q}_{2}$ & $\mathrm{Q}_{3}$ \\
State sequence $(\mathrm{Q}):$ & 1 & 2 & 3 \\
State transition probability, $\mathrm{a}_{\mathrm{ij}}:$ & 0.5 & 0.5 & 0.5 \\
Observation probabilities, $\mathrm{b}_{\mathrm{i}}(\mathrm{k}):$ & 0.26 & 0.98 & 0.98 \\
\hline
\end{tabular}

Total probability for Kayes, $\mathrm{P}(\mathrm{X} 1 \mathrm{Q})=0.03121$ 


\subsection{Result}

The highest score is obtained from Manjur, hence the unknown speaker was Manjur.

\section{CONCLUSION}

In this work the system has been trained for each speaker for same word 'CSE' which the speaker utters for three times. Then particular speaker are specified by comparing each registered speaker in the training phase. In the training phase actually a reference model is built for a particular speaker and compared that stored reference model against the input speech by evaluating scores using HMM and speaker identification with the best score is done. The system developed is speaker and text dependent and moderately tolerant to background noise hence it is a very efficient system. Due to dynamic nature of the speech signals, current speaker recognition systems produce reasonable results, but still lack the necessary performance if they are to be used the general public. The variability in speech is mainly caused by the length of the vocal track, varying pitch and speaking rate as well as different accents and speaking styles This paper challenged this problem and the results obtained did not only improve the identification rate but also improved the reliability of the SI system. The system uses the MFCC feature extraction technique and HMM for evaluating the scores which proved to be a great success. The experiment is carried out in noise free environment. The system correctly identified the speaker trained for a particular word by comparing the input speech for that word against the stored reference model for that word.

\section{REFERENCES}

[1] Speaker Verification using Vector Quantization and Hidden Markov Model. Mohd Zaizu Ilyas, Member, IEEE, Salina Abdul Samad, Senior Member, IEEE, Aini Hussain, Member, IEEE and Khairul Anuar Ishak, Member, IEEE, "The 5th Student Conference on Research and Development -SCOReD 2007 11-12 December 2007, Malaysia.

[2] MFCC and its applications in speaker recognition, Vibha Tiwari. Deptt. of Electronics Engg., Gyan Ganga Institute of Technology and Management, Bhopal, (MP) INDIA (Received 5 Nov., 2009, Accepted 10 Feb., 2010), "International Journal on Emerging Technologies 1(1): 19-22(2010) ISSN : 0975-8364.
[3] Text-Independent Speaker Identification Using Hidden Markov Model Sayed Jaafer Abdallah, Izzeldin Mohamed Osman, Mohamed Elhafiz Mustafa, College of Computer Science and Information Technology Sudan University of Science and Technology, "World of Computer Science and Information Technology Journal (WCSIT) ISSN: 22210741 Vol. 2, No. 6, 203-208, 2012 Khartoum, Sudan.

[4] SPEAKER IDENTIFICATION USING MEL FREQUENCY CEPSTRAL COEFFICIENTS, Md. Rashidul Hasan, Mustafa Jamil, Md. Golam Rabbani Md. Saifur Rahman, Electrical and Electronic Engineering, Bangladesh University of Engineering and Technology, "Dhaka-1000, 3rd International Conference on Electrical \& Computer Engineering ICECE 2004, 28-30 December 2004, Dhaka, Bangladesh.

[5] Speaker Recognition Using MFCC Front End Analysis and VQ Modeling Technique for Hindi Words using MATLAB, Nitisha M.Tech. (pursuing) Hindu College of engineering Sonipat, Haryana India, Ahu Bansal Assistant professor Hindu College of engineering Sonipat, Haryana, India. "International Journal of Computer Applications (0975 - 8887) Volume 45No.24, May 2012.

[6] HMM Speaker Identification Using Linear and Nonlinear Merging Techniques Unathi Mahola, Fulufhelo V. Nelwamondo, Tshilidzi Marwala School of Electrical and information Engineering University of the Witwatersrand, Johannesburg, South Africa.

[7] J.M. Naik, "Speaker Verification: A Tutorial", IEEE Communication Magazine, January 1990, pp. 42-48.

[8] J.P. Campbell, "Speaker Recognition: A tutorial “, Proc. of the IEEE, Vol. 85, No. 9, September 1997, pp. 1437 1462 .

[9] L.R. Rabiner, "A Tutorial on Hidden Markov Models and Selected Application in Speech Recognition", Proceeding of The IEEE, Vol.77, No.2, February 1989.

[10] L.R. Rabiner and B.H. Juang, Fundamental of Speech Recognition, Prentice Hall, New Jersey, 1993.

[11] National Science and Technology Council (NTSC), "Speaker Recognition", 2006. [Online]. Available: http://www.biometricscatalog.org/NSTCSubcommittee/ Documents/Speaker\%20Recognition.pdf $\left[\begin{array}{ll}7 & \text { August }\end{array}\right.$ 2006]. 